\title{
EDITORIAL
}

\section{GERIATRIC MEDICINE IN ITALY IN THE TIME OF COVID-19}

\author{
M. CESARI ${ }^{1,2}$, M. PROIETTI ${ }^{1,2,3}$ \\ 1. Geriatric Unit, Fondazione IRCCS Ca' Granda Ospedale Maggiore Policlinico, Milan, Italy; 2. Department of Clinical Sciences and Community Health, University of Milan, Milan,

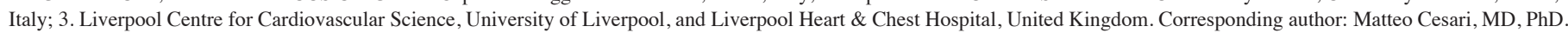 \\ Geriatric Unit, Fondazione IRCCS Ca’ Granda Ospedale Maggiore Policlinico; Via Pace 9, 20122 Milan, Italy. Email: macesari@ gmail.com. Twitter: @macesari
}

On March 11, 2020, the World Health Organization declared the SARS-CoV-2 outbreak a pandemic (1). To date, the number of coronavirus cases has passed 400,000 worldwide, and more than 18,000 persons have died. After China, Italy is the country that has been most violently hit by the COVID-19 tsunami. With 69,176 cases and 6,820 deaths (updated on March 24th, 2020), the case fatality rate due to COVID-19 in Italy is one of the highest in the world (about 9.8\%), well above what has been recorded in China (2). In particular, the Lombardy Region (with its cities of Bergamo, Brescia, Lodi, and Milan) is paying the heaviest toll to the coronavirus in terms of fatalities (3). The vast majority of deaths is occurring in older persons, a fact that can only be partially explained by the older age of the Italian population. In fact, although current data indicate that persons aged 70 years and older contribute to about the $85 \%$ of the death events in Italy, it cannot be overlooked the fact that Japan has substantially smaller figures despite being the oldest country in the world. It is likely that the strategies adopted for testing the population as well as the robustness of the healthcare system may play a much more relevant role than age in the explanation of the scenario.

Unfortunately, numbers can only provide a partial description of what is happening, and probably underestimate the ongoing tragedy (3). Right in these days, the international media have broadcasted worldwide the dramatic column of military trucks leaving the city of Bergamo laden with the coffins of many patients died of COVID-19. People cannot even celebrate the funeral of their relatives who died isolated in COVID-19 facilities. Schools, universities, activities... all of them have been closed; the entire country is locked down since March 21st. The worldwide web is full of videos showing how healthcare professionals are today working around the clock, often with limited resources.

The limitation of resources has indeed been a major issue, especially during the very first days of the crisis when there still was a certain dose of unpreparedness and surprise in front of the magnitude of what was happening. Low number of ventilators, saturation of beds in intensive care units, shortage of personal protective equipment, overcrowding emergency departments, inability to adequately isolate suspected cases... have been the most urgent and critical problems faced by clinicians, especially at the beginning. It is thus not surprising why about $10 \%$ of the persons infected by the SARS-CoV-2 is today constituted by physicians and nurses. Working in such an emergency to counteract the enormity of what was arriving unavoidably led to healthcare professionals falling sick (and sometimes even die) under the coronavirus. The consequent reduction of the healthcare professionals due to COVID19 has been threatening the sustainability of the system. To overcome this critical moment, many healthcare professionals from everywhere in the world have answered to the call for help, and converged to Italy to put their expertise at disposal of our community. To keep the healthcare machine running and support the colleagues overwhelmed in the management of COVID patients, there have been pediatricians working with older patients, or surgeons taking care of internal medicine issues... Everyone is giving what he/she can.

How is the life of a geriatrician in such an apocalyptic scenario? Geriatricians are sadly used to the fact that our hedonistic society often finds no place for older people (4). In normal times, geriatricians regularly battle for guaranteeing the proper care to persons who are otherwise excluded from interventions just because they are "old". Geriatric medicine has produced substantial evidence showing that frail older persons require adaptations in the clinical approach, and that the environment plays a critical role for the wellbeing of the aging individual $(5,6)$. Well...everything we know has simply been wiped away by this period of crisis we are living. It can be understandable that the focus is today exclusively on the SARS$\mathrm{CoV}-2$, and the hospitals are primarily acting for defeating it. Nothing else seems important in front of the enormity of the crisis. The differences across specialties have been cancelled because SARS-CoV-2 is everywhere, probably more diffused than what is generally thought or objectively measured by tests. The only priority in the clinical setting is to isolate COVID patients (or suspect cases) as soon as possible, and offer prompt respiratory support when symptoms appear and start to dramatically worsen. The coronavirus has deleted everything that can be felt superfluous, redundant, and/or unnecessary. Unfortunately, geriatric medicine did not start the coronavirus crisis with solid roots. It has traditionally been considered a "poor specialty", always struggling for recognition. It was indeed not in the best position compared to other disciplines to protect the results it has been accomplishing over the past decades for the care of frail older persons. Furthermore, it has never been a winning ticket for geriatrics to specifically serve those frail older persons, often at the margin of the society. Under this scenario, it has not taken a lot before everything 


\section{GERIATRIC MEDICINE IN ITALY IN THE TIME OF COVID-19}

related to geriatrics was forgotten in the name of the battle against the coronavirus. Ubi major minor cessat... Is this right? Is this the way things should go? Of course, not! The fact that the emergency has forced drastic (and sometimes cynical) decisions does not justify the loss of attention to the person's values. Listening a physician stating "Luckily, in our hospital no one younger than 60 years has died" provides a clear taste of the well-known ageism of our society. This kind of attitude was before (more or less) hidden behind a façade of politically correct, but is receiving now a sort of legitimation in front of the dramatic emergency. When media have been underlining that older persons with comorbidities were the most likely to die, a relief breath was made by many (as they had no older relatives, or as they will not be old as well one day!). Nevertheless, geriatricians are also to blame for the failure we experience today. We have lost ourselves and wasted time running after futile controversies, missing opportunities for contaminating other disciplines with the correct principles of geriatric medicine. We probably remained closed inside our ivory towers, and left others design their (inadequate) solutions for our patients (7).

The costs of the coronavirus for older persons are and will likely remain underestimated. We can count deaths events occurring in individuals resulting positive to the test. However, these figures do not consider that:

1) Tests have not been routinely available, especially for older persons. For example, long term care facilities and primary care physicians cannot yet easily perform the test for the identification of SARS-CoV-2.

2) The test is limited by a relatively high number of false negative results. Clinicians are quite confident that many patients died of COVID despite not officially being diagnosed for it. Although these patients could still be managed with all the precautions as they were COVID, they have still been excluded from the statistics. There has been a patient in our hospital who resulted three times negative at the nasopharyngeal swab, before finally being found positive at the Broncho-Alveolar Lavage.

3) The already fragile integration of care services completely collapsed under the coronavirus diffusion. Hospitals closed within themselves for limiting the diffusion of the virus, while post-acute/long-term care facilities reduced admissions being afraid of a possible contamination arriving from outside. Primary care physicians have been paying a huge tool (even with their lives), finding themselves isolated in the community with no means and no support from the hospitals. Many older persons (with their chronic conditions and care needs) remained isolated after the SARS-CoV-2 outbreak The consequences of this are not part of the standard COVID narrative...

4) In a situation of constant emergency, it is almost impossible to apply many interventions against geriatric conditions across clinical settings. Physical therapists for mobilization of the older patient, avoidance of stressors for prevention of delirium, social contacts for re-orientation of the person, teamwork for the design of person-tailored interventions... all this does not exist anymore. There is no time, no personnel, no resources for supporting the good practice of geriatric medicine. The same human interaction between the patient and his/her physician is lost behind the burdening personal protective equipment in COVID-19 facilities. Are we redundant? We prefer to think that we have not yet been able to communicate how certain care standards solicited by geriatricians are necessary for practicing a modern medicine.

What will remain after the SARS-CoV-2 will be defeated? We will count our deaths. We will probably take some time for thinking at our acts and decisions. We will surely see many errors, but this will give us a unique opportunity for improvement. After all, Albert Einstein said "The crisis is the greatest blessing for people and nations, because the crisis brings progress". Hopefully, we will learn from our mistakes and contribute for a better society... a society able to give more value to persons, independently of their age.

Disclosures: No conflict of interest to declare.

\section{References}

1. WHO Director-General's opening remarks at the media briefing on COVID-19 - 11 March 2020. https://www.who.int/dg/speeches/detail/who-director-general-sopening-remarks-at-the-media-briefing-on-covid-19---11-march-2020. Accessed March 23, 2020.

2. Onder G, Rezza G, Brusaferro S. Case-Fatality Rate and Characteristics of Patients Dying in Relation to COVID-19 in Italy. JAMA. March 2020. doi:10.1001/ jama.2020.4683.

3. Nacoti M, Ciocca A, Giupponi A, et al. At the Epicenter of the Covid-19 Pandemic and Humanitarian Crises in Italy: Changing Perspectives on Preparation and Mitigation. N Engl J Med Catal. 2020:5. doi:10.1056/CAT.20.0080.

4. Wright JL. The Courage to Be a Geriatrician. J Am Geriatr Soc. 2014;62(6):1139 1141. doi:10.1111/jgs.12837.

5. Tinetti M, Fried T. The end of the disease era. Am J Med. 2004;116(3):179-185.

6. Lim W, Wong S, Leong I, Choo P, Pang W. Forging a Frailty-Ready Healthcare System to Meet Population Ageing. Int J Environ Res Public Health. 2017;14(12):1448. doi:10.3390/ijerph14121448.

7. Cesari M, Marzetti E, Thiem U, et al. The geriatric management of frailty as paradigm of "The end of the disease era". Eur J Intern Med. 2016;31:11-14. doi:10.1016/j.ejim.2016.03.005. 